\title{
GENERALIZED COLLECTIVE MODES IN LIQUID CESIUM
}

\author{
T. Bryk, I. Mryglod \\ Institute for Condensed Matter Physics, National Academy of Sciences of Ukraine \\ 1 Svientsitskii St., Lviv, UA-79011, Ukraine
}

(Received January 4, 2003; received in final form September 5, 2003)

\begin{abstract}
The spectrum of collective excitations of liquid Cs at $308 \mathrm{~K}$ is investigated within the ninevariable approximation of generalized collective mode approach. An original analysis of spectra is performed to establish the physical meaning as well as the origin of all the propagating and relaxing collective modes found. Two branches of low- and high-frequency propagating excitations are identified with the heat waves. It is shown that a kinetic relaxing mode, caused by density fluctuations, defines almost completely the shape of 'density-density' time correlation function for wavenumbers close to the main peak position of the static structure factor. Amplitudes of mode contributions to the 'density-density' and 'heat density-heat density' time correlation functions are studied in detail.
\end{abstract}

Key words: generalized hydrodynamics, collective excitations, liquid metals, time correlation functions, structure relaxation in liquids.

PACS number(s): 05.20.Jj, 61.20.Ja, 66.10.Cb

\section{INTRODUCTION}

Liquid cesium is one of the most simple and most studied representatives of a wide family of alkali metallic liquids with well-defined collective excitations, which are observable on dynamical structure factor $S(k, \omega)(k$ and $\omega$ being wavenumber and frequency, respectively) in a broad range of wavenumbers $k$ (up to $k \sim 0.8 k_{\mathrm{p}}$ [1] in the case of cesium, where $k_{\mathrm{p}}$ is the position of the first maximum of the static structure factor $S(k)$ ). This feature of liquid alkali metals makes a significant difference with other simple liquids, for instance with dense Lennard-Jones systems, and has attached intensive theoretical and experimental investigations. Neutron scattering experiments [2,3], performed on liquid Cs just above the melting point, have shown that: (i) the well-visible peak at $\omega \neq 0$ exists in $S(k, \omega)$ for $k<1.2 \AA^{-1}$; (ii) at $k \approx k_{\mathrm{p}}=1.4 \AA^{-1}$ the well-known de Gennes narrowing, governed by a cooperative-diffusion-like relaxation process, is observed; (iii) for $1.8 \AA^{-1}<k<2.0 \AA^{-1}$ the existence of propagating modes in liquid Cs appears as a shoulder in $S(k, \omega)$. In addition to these features it was found that the damping coefficient of observed propagating modes increases almost linearly in the $k$ region covered in the experiment, and a clear enhancement over the sound velocity is seen in the dispersion of density fluctuations for small wavenumbers $k$. This allowed to assume that the clear evidence of nonhydrodynamic propagation is observed in liquid cesium near the melting point and the further experimental and theoretical attempts to explain the features observed are needed $[2,3]$.

Cesium near the melting point was studied rather intensively by computer simulations [4-6]. Kambayashi and Kahl performed [4] a molecular dynamics study on liquid Cs with two-body potentals derived from Ashcroft empty-core pseudopotentials, and found very good agreement between the MD results and the experimental data for both static and dynamic structure factors. It was also found no sufficient evidence for existence of propagating sound modes beyond the main peak in $S(k)$, although the authors correctly mentioned that the absence of distinct peaks in $S(k, \omega)$ does not imply the absence of propagating sound modes. An important consequence of the molecular dynamics simulations, performed in Refs. [5,6] for models of liquid $\mathrm{Na}, \mathrm{K}, \mathrm{Rb}$ and $\mathrm{Cs}$ near the melting point with the pair potential proposed by Price, Singwi and Tosi [7], is that both the equilibrium and the timedependent correlations can be cast in a properly scaled form, which is in a very good approximation the same for all the alkali liquid metals. Within this 'unitary description' of the 'classical' alkali metals it was shown that the presence of well-defined propagating modes at relatively large wavenumbers (up to $k \leq 3 k_{\mathrm{p}} / 4$ ) appears to be peculiar to them near the melting point. Note that the scaling idea used in Ref. [5] is somewhat similar to that of Lennard-Jones potentials for inert gases, and this indicates strongly that the different dynamic behaviour of Lennard-Jones-like systems and liquid-alkali metals is mainly attributed to the different softness of the potential cores. Such a conclusion was numerically verified $[8,9]$ in molecular dynamics simulations, and it was shown that the Brillouin peaks in $S(k, \omega)$ are more pronounced and persist up to larger $k$ for softer potentials.

In the theoretical analysis of molecular dynamics data [4-6] the dynamical properties of liquid Cs were mainly considered (see also $[10,11]$ ) within the hydrodynamic treatment, viscoelastic or simplified mode-coupling theories. To our knowledge the first attempt to treat a liquid metal within the generalized collective mode (GCM) approach was reported in Ref. [12]. Herein, the spectrum of generalized longitudinal collective excitations in liquid Cs was obtained within the simplest non-trivial fivevariable scheme, and very good agreement with the MD results for the 'density-density' time correlation function in a wide range of wavenumbers $k$ was found. Considering the higher order mode approximations, in [13] the dynamics of transverse fluctuations in Cs was studied with 
the main attention focused on the properties of transverse excitations and $(k, \omega)$-dependent shear viscosity.

The concept of GCM was initially suggested in [14] to investigate dynamical properties of a simple LennardJones liquid in a wide range of wavevectors. The main idea of this method was to extend the basis set of dynamical variables by taking in addition to the hydrodynamic ones their time derivatives, which were supposed to describe short-time processes in liquids. The time evolution of these 'extended' variables was obtained in molecular dynamics (MD) simulations in order to evaluate relevant time correlation functions and static averages. All static averages and some fitting parameters were used then to estimate the matrix elements of secular equation derived from the generalized Langevin equation. In [15] this method of generalized collective modes (GCM) was modified into a parameter-free approach (see also [16]) and has advanced later in [17] to a high-variable approximation with up to three first time derivatives of the hydrodynamic variables. In general, the basis set of $N_{v}$ dynamical variables generates $N_{v} \times N_{v}$ secular equation and results in $N_{v}$ generalized collective modes (eigenvalues). The GCM method proved to be very useful for investigation of spectra of collective excitations in LennardJones pure liquids [14-17], a semimetallic liquid Bi [18], liquid water [19], 'fast-sound' mixture $\mathrm{He}_{65} \mathrm{Ne}_{35}$ [20-22], Lennard-Jones binary mixture [23-25], liquid glassforming metallic alloy $\mathrm{Mg}_{70} \mathrm{Zn}_{30}$ [24-26], and metallic molten alloy $\mathrm{Li}_{4} \mathrm{~Pb}[24]$.

In the hydrodynamic limit $(k \rightarrow 0, \omega \rightarrow 0)$ the collective mode spectrum can be studied analytically using three-mode treatment [27-29]. For longitudinal dynamics in pure liquids there exist three conserved dynamical variables (density of particles $\hat{n}(k, t)$, density of longitudinal current $\hat{J}_{l}(k, t)$, and energy density $\left.\hat{e}(k, t)\right)$ for which the local conservation laws are satisfied. The solution of hydrodynamic equations for pure liquids is wellknown: three collective modes completely determine the longitudinal dynamics of pure liquid in long-wavelength limit. They are the two sound waves propagating in opposite directions (a pair of complex-conjugated eigenvalues) and one relaxing thermodiffusive mode (a purely real eigenvalue).

Among $N_{v}$ eigenvalues the lowest ones (three modes in the case of pure liquids, and four ones for binary systems) always correspond in the region of small wavenumbers to the hydrodynamic excitations, predicted by the linear hydrodynamics. All the other eigenvalues are called kinetic modes and correspond to the processes of shorttime scale. These modes cannot be obtained within the standard hydrodynamic treatment. Namely the kinetic modes are responsible for the 'fast sound' phenomenon in binary liquids [21]. Also, optic-like excitations in manycomponent liquids are in fact the kinetic modes. However, no attention was paid to the possibility to observe kinetic modes in collective dynamics of pure liquids. Even, the origin of kinetic modes for pure systems is not known a priori. Therefore, the theoretical study of generalized mode spectra with special attention to the origin of modes formation in pure metals and semimetals would be of great interest.

The first attempt to treat a liquid metal within the GCM approach was reported in Ref. [12]. The spectrum of liquid Cs was obtained within the simplest nonhydrodynamic five-variable treatment. However, the origin of kinetic collective modes was not studied herein. The next investigation of transverse dynamics of liquid Cs [13] was already performed within the high-variable approximation and was focused on the study of $(k, \omega)$ dependent shear viscosity of a liquid metal. The origin of kinetic collective modes in a liquid semimetallic Bi was considered in our recent papers [18]. The most recent study of both kinetic propagating and relaxing collective modes for two temperatures within the high-variable approximation of GCM method has been performed for liquid $\mathrm{Pb}[30]$.

The goal of this study is: (i) to estimate the spectrum of generalized collective excitations for a liquid Cs within the high-variable approximation of the GCM approach; (ii) to establish the origin of longitudinal kinetic propagating and relaxing collective modes found in the spectrum; and, (iii) to focus on the contributions of different collective modes to various time correlation functions.

The paper is organized as follows: in Sec. II we briefly describe the methodology of our calculations; the results for spectrum of generalized collective excitations as well as for the separated mode contributions to different time correlation functions are reported in Sec. III, and conclusions are given in Sec. IV.

\section{DETAILS OF CALCULATIONS}

For the generalized treatment of longitudinal dynamics within the GCM method we accept the nine-variable basis set consisted of the following operators:

$$
\mathbf{A}^{(9)}(k, t)=\left\{A_{i}(k, t)\right\}=\left\{n(k, t), J_{l}(k, t), e(k, t), \dot{J}_{l}(k, t), \dot{e}(k, t), \ddot{J}_{l}(k, t), \ddot{e}(k, t), \dddot{J}_{l}(k, t), \dddot{e}(k, t)\right\},
$$

where the dots denote the order of the time derivative of relevant operator $A_{i}$. This basis set of dynamical variables was used to generate the eigenvalue problem for generalized hydrodynamic matrix $\mathbf{T}(k)$ (see [16-18]).
Complex-conjugated and purely real eigenvalues $z_{\alpha}(k)$ of the generalized hydrodynamic matrix form the spectrum of collective excitations.

In general case, for the basis set of $N_{v}$ dynamic vari- 
ables, all the time correlation functions, constructed on the variables $\left\{A_{i}\right\}$, can be expressed in the GCM approach as a weighted sum of $N_{v}$ mode contributions, namely:

$$
F_{i j}^{\mathrm{GCM}}(k, t)=\sum_{\alpha=1}^{N_{v}} G_{i j}^{\alpha}(k) e^{-z_{\alpha}(k) t}
$$

where $G_{i j}^{\alpha}(k)$ is the weight coefficient of $\alpha$ th mode contribution to the time correlation function $F_{i j}(k, t)$. The weight coefficients $G_{i j}^{\alpha}(k)$ can be expressed via the relevant eigenvectors associated with the $\alpha$ th eigenvalue. In contrast to purely hydrodynamic treatment (expressions with three mode contributions), functions (2) reproduce explicitly the first $(2 S+1)$ frequency moments of relevant spectral function $F_{n n}^{\mathrm{GCM}}(k, \omega), F_{J J}^{\mathrm{GCM}}(k, \omega)$, and $F_{e e}^{\mathrm{GCM}}(k, \omega)$, where $S$ is the maximum number of dots of particular operator $A_{i}$ taken in the basis set. Moreover, because of the relation $\dot{n}(k, t) \sim J_{l}(k, t)$, for the 'densitydensity' time correlation function $F_{n n}^{\mathrm{GCM}}(k, \omega)$ the first $(2 S+3)$ frequency moments coincide with the genuine ones. Thus, in the particular case of the basis set (1), the calculated function $F_{n n}^{\mathrm{GCM}}(k, \omega)$ should contain itself the first nine exact frequency moments (including zeroth one), while the GCM 'energy-energy' function does seven ones.

The expression for the Laplace-transforms, which follows from (2),

$$
\tilde{F}_{i j}^{\mathrm{GCM}}(k, z)=\sum_{\alpha=1}^{N_{v}} \frac{G_{i j}^{\alpha}(k)}{z+z_{\alpha}(k)}
$$

allows us to study in detail the mode contributions to the dynamical structure factor or any other spectral function of interest. Note that in (2) and (3) the weight coefficients $G_{i j}^{\alpha}(k)$ are in general the complex functions, so that taking into account symmetrical properties of the amplitudes and the assumption that among $N_{v}$ eigenvalues there are $N_{\text {pr }}$ pairs of complex-conjugated eigenvalues $z_{\alpha}^{ \pm}(k)$ (or propagating modes) with

$$
z_{\alpha}^{ \pm}(k)= \pm i \omega_{\alpha}(k)+\sigma_{\alpha}(k), \quad \alpha=1,2, \ldots, N_{\mathrm{pr}}
$$

and $N_{\mathrm{rl}}$ purely real ones (or relaxing modes) with $z_{\alpha}(k)=d_{\alpha}(k)$, one can rewrite $(2)$ in a widely accepted form

$$
F_{i j}^{\mathrm{GCM}}(k, t)=\sum_{r=1}^{N_{\mathrm{rl}}} A_{i j}^{r}(k) e^{-d_{r}(k) t}+\sum_{p=1}^{N_{\mathrm{pr}}}\left\{B_{i j}^{p}(k) \cos \left[\omega_{p}(k) t\right]+C_{i j}^{p}(k) \sin \left[\omega_{p}(k) t\right]\right\} e^{-\sigma_{p}(k) t}
$$

where all the amplitudes $A^{r}, B^{p}$ and $C^{p}$ are the functions of $k$ only. We will call the terms with amplitudes $B^{p}$ and $C^{p}$, originated by propagating modes, as symmetric and asymmetric ones, respectively. Obviously, that the equality

$$
\sum_{r=1}^{N_{\mathrm{rl}}} A_{i j}^{r}(k)+\sum_{p=1}^{N_{\mathrm{pr}}} B_{i j}^{p}(k)=F_{i j}(k, t=0)
$$

should be satisfied giving in fact the zeroth frequency moment of $F_{i j}(k, \omega)$. The expression for the higher order frequency moments can be derived from (2) straightforward by considering the time derivatives at $t=0$. By taking the Fourier transform of the expression (5) one can study the separated mode contributions into spectral functions $F_{i j}(k, \omega)$, which are formed by: $N_{\text {rl }}$ central Lorentzians, $N_{\text {pr }}$ noncentral Lorentzians (symmetric contributions), and $N_{\mathrm{pr}}$ non-Lorentzian corrections (asymmetric contributions), respectively. It is well-known that the functions $F_{i j}(k, \omega)$ are simply related to the corresponding Laplace transforms $\tilde{F}_{i j}(k, z)$,

$$
F_{i j}(k, \omega)=\frac{1}{\pi} \operatorname{Re} \tilde{F}_{i j}(k, z),
$$

so that the expression (3) is very useful in this respect.

To obtain spectra of collective excitations in a liquid within the GCM method, one has to estimate the elements of generalized hydrodynamic matrix $\mathbf{T}(k)$ first. The information about static correlation functions needed can easily be extracted from the molecular dynamics simulations for the system considered. Solving then the eigenvalue problem for matrix $\mathbf{T}(k)$ at each $k$-value sampled in MD, we obtain the set of $k$-dependent eigenvalues which form the generalized collective mode spectrum. Eigenvectors, estimated in such an approach, allow us to obtain the weight coefficients, giving the information about the separated mode contributions to time correlation functions considered. In our subsequent study we follows along this general scheme, described in details, for instance, in Ref. [16, 18,30,31]. It should be emphasized that the results, to be obtained in such an approach, describe the $N_{v} \times N_{v}$ set of time correlation functions in self-consistent way, giving all the needed information about maximum positions in the spectral functions, the height and width the origin of collective excitations.

The MD simulations were performed in standard microcanonical ensemble for liquid Cs with mass density $\rho=1832.1 \mathrm{~kg} \mathrm{~m}^{-3}$ at average temperature $308 \mathrm{~K}$. We consider a finite system of 1000 particles interacting through oscillating potential $\Phi_{i j}(r)$ at constant volume 
$V=L^{3}$. The smallest wavenumber reached in our MD study is $k_{\min }=0.1272 \AA^{-1}$. Time evolution of hydrodynamic variables and their time derivatives was observed during the production run over $3 \cdot 10^{5}$ steps. The effective two-body potential was taken from [4]. This potential reproduced very nicely the experimental static structure factor of liquid Cs near the melting point. The following energy, length and time scales $\varepsilon=k_{\mathrm{B}} T, \sigma=k_{\min }^{-1}$, $\tau=\sigma(m / \varepsilon)^{1 / 2}=5.6626$ ps are used below for the reduction of dimensional quantities.

\section{RESULTS AND DISCUSSION}

\section{A. Spectrum of collective excitations}

Results, obtained for eigenvalues of the generalized hydrodynamic matrix $\mathbf{T}(k)$, generated on the basis set (1), are shown in Figures 1 and 2. As the functions of $k$ they form the spectrum of collective excitations. It is seen, that for $k<2.2 \AA^{-1}$ the spectrum consists of three branches of propagating modes (three pairs of complex conjugated eigenvalues $z_{\alpha}^{ \pm}(k)$ with $\alpha=1,2,3$ in Figures $1 \mathrm{a}$ and $1 \mathrm{~b})$. In this $k$-region there exist also three relaxing modes (see Figure 2) with purely real eigenvalues $z_{i}(k)=d_{i}(k)$ with $i=1,2,3$, so that the total number of collective modes is equal to nine as it should be for the nine-variable set (1).

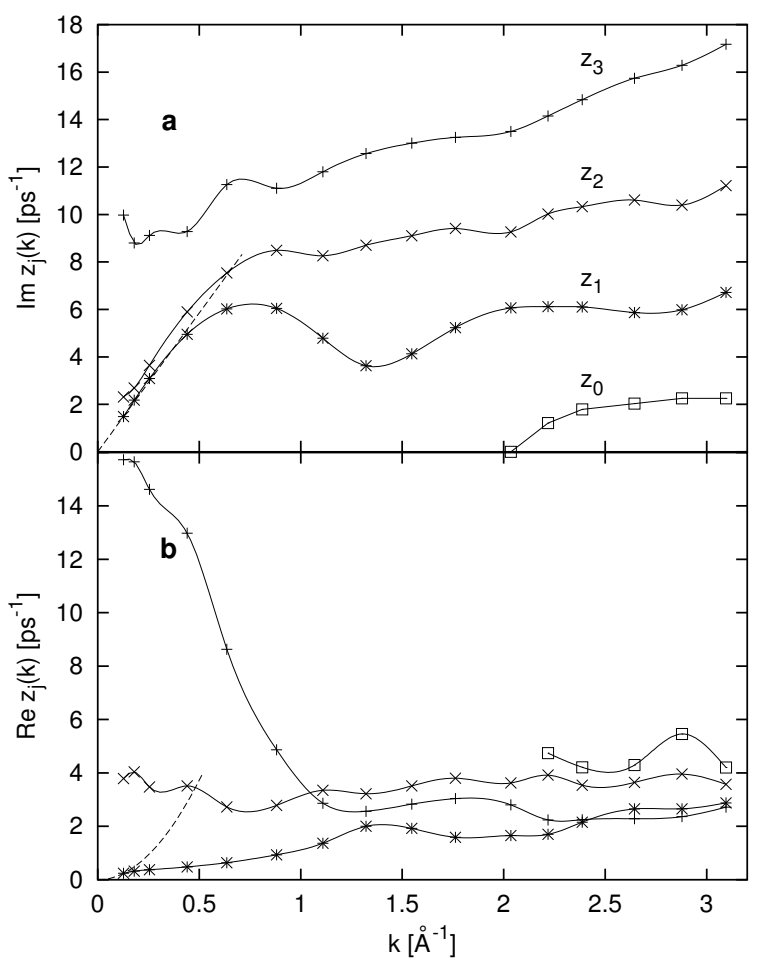

Fig. 1. Spectrum of propagating collective excitations of a liquid $\mathrm{Cs}$ at $308 \mathrm{~K}$, obtained for the nine-variable basis set $\mathbf{A}^{(9)}(k, t)$. Imaginary and real parts of eigenvalues are shown in frames (a) and (b), respectively. Asymptotic hydrodynamic behaviour for the sound excitations is shown by dashed lines. Solid lines are the spline interpolation between the MD data.
In Figure 1a one can see the emergence of the fourth pair of propagating modes at $k=k_{0} \simeq 2.2 \AA^{-1}$, which is well observed also for larger wavenumbers. The real part of the corresponding eigenvalues $z_{0}^{ \pm}(k)$ defines the damping coefficient, which is the highest one among all complex eigenvalues (see Figure 1b for $k>k_{0}$ ). For smaller $k$-values the propagating modes $z_{0}^{ \pm}(k)$ disappear in so-called propagating gap, creating instead the pair of purely real eigenvalues. Thus, in the region $k>2.2 \AA^{-1}$ there exists only a single relaxing mode within the ninevariable GCM treatment. We note that almost the same picture for generalized collective spectrum was observed in our previous studies of a LJ liquid [17], metallic liquid $\mathrm{Pb}$ [30], and semimetallic liquid $\mathrm{Bi}$ [18]. The main difference between these cases is in the mutual disposition of propagating modes and in the value of $k_{0}$, where the branch $z_{0}^{ \pm}(k)$ disappears. For instance, in the case of liquid $\mathrm{Bi}[18]$ we have not found the branch $z_{0}^{ \pm}(k)$ for $k<3 \AA^{-1}$. For more detailed discussion of the origin of low-frequency kinetic propagating excitations we refer the readers to the Refs. $[18,30]$, while in this study we will mainly focus on the region $k<1.8 \AA^{-1}$ and especially on the origin of kinetic relaxing modes.

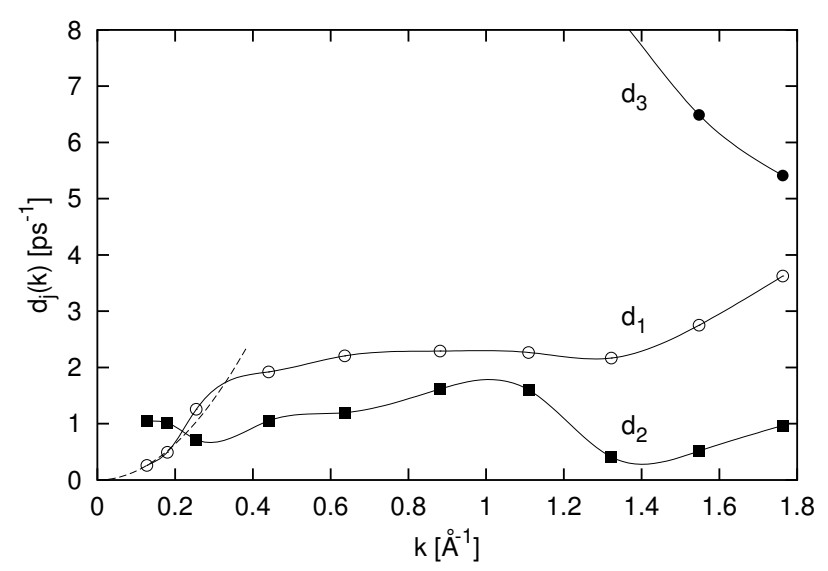

Fig. 2. Purely real eigenvalues of a liquid Cs at $308 \mathrm{~K}$, obtained for the nine-variable basis set $\mathbf{A}^{(9)}(k, t)$. Asymptotic hydrodynamic behaviour for the thermodiffusive modes is shown by dashed lines. Solid lines are the spline interpolation between the MD data.

From the behaviour of complex eigenvalues at $k \rightarrow 0$ one can estimate, that the lowest pair of propagating modes $z_{1}^{ \pm}(k)$ corresponds to the generalized sound excitations with linear dispersion $\omega_{s}(k)$ in the small- $k$ region:

$$
z_{1}^{ \pm}(k) \simeq \Gamma k^{2} \pm i c_{s} k, \quad k \rightarrow 0 .
$$

It is seen in Figure 1a that in the vicinity of main peak of the static structure factor $S(k)$, located for this thermodynamic point of liquid Cs at $k_{\mathrm{p}}=1.4 \AA^{-1}$ (see [4,12]), the branch $z_{1}^{ \pm}(k)$ exhibits a wide minimum. We note that when $k \sim k_{\mathrm{p}}$ the damping of sound modes is still 
not sufficiently larger (see Figure 1b) than their frequency $\left(\sigma_{s}<\omega_{s}\right)$, so that these propagating excitations are well defined in that region.

The straight dashed line in the upper frame of Figure 1a illustrates, that the lowest branch $z_{1}^{ \pm}(k)$ has a 'positive dispersion', that is in complete agreement with the results of previous studies $[2-4,12]$. From the slope of this line the sound velocity in liquid Cs can easily be estimated, and we found $c_{s} \simeq 1170 \mathrm{~m} / \mathrm{s}$, that is nearly the same value as obtained within the five-variable treatment [12]. It should be noted that this value differs rather significantly from the experimental result [34], which, otherwise, is in a good agreement with estimate following from the well-known formula for adiabatic sound velocity $c_{s}=\left[k_{\mathrm{B}} T \gamma / S(0) m\right]^{1 / 2} \simeq 970 \mathrm{~m} / \mathrm{s}$. To understand such a difference, which is difficult to explain completely due to anomalous dispersion caused by the hydrodynamic mode coupling effects $[32,33]$, one can agree with the view-point proposed in [3]. The real part of $z_{1}^{ \pm}(k)$, which defines the damping (or inverse time of life) of generalized sound excitations, in hydrodynamic limit must be the function of $k^{2}$ with $\Gamma$ being the sound attenuation coefficient. From the smallest $k$-point we have estimated $\Gamma$ to be $1.49 \cdot 10^{-7} \mathrm{~m}^{2} / \mathrm{s}$. Beyond the hydrodynamic region the short-time effects become more important and they can change the dependence (7). For the case of real parts of eigenvalues $z_{1}^{ \pm}(k)$ the departure from hydrodynamic parabolic form (shown by dashed line in Fig. 1b) displays negative dispersion. Qualitatively this is in agreement with predictions of mode coupling theory [32,33], but taking into account the results of [3], other mechanisms of such a behaviour can be considered as well. Note that beyond the hydrodynamic region $\left(k>0.2 \AA^{-1}\right.$ and up to $k \simeq 1.2 \AA^{-1}$ ) the damping coefficient $\sigma_{s}(k)$ could be well fitted by a linear function of $k$ what was noted in [3]. In particular, this indicates strongly that the viscoelastic mechanism of Maxwell-like relaxation might be very important beyond the hydrodynamic region.

Another hydrodynamic eigenvalue, which is purely real one and corresponds to the thermodiffusive mode, has to behave according to predictions of the linear hydrodynamics as follows

$$
d_{1}(k) \simeq D_{T} k^{2}, \quad k \rightarrow 0
$$

with $D_{T}$ being the thermodiffusion coefficient. As one can judge from the Figure 2 (dashed line), the $k$-dependence of the lowest lying real eigenvalue in the small $k$ region $\left(k<0.2 \AA^{-1}\right)$ behaves like (8). From the smallest $k$-point we have estimated the value $D_{T}=1.60 \cdot 10^{-7} \mathrm{~m}^{2} / \mathrm{s}$. It is seen in Figure 2, that in fact two purely real eigenvalues $d_{1}(k)$ and $d_{2}(k)$ are very close each other for $k<1.2 \AA^{-1}$, so that it is rather difficult to distinguish a priori their $k$-dependence. The way we have separated in this study the relaxing modes $d_{1}(k)$ and $d_{2}(k)$ in the Figure 2 will be explained in the next subsection.

The pair of propagating excitations $z_{1}^{ \pm}(k)$ and thermodiffusive mode $d_{1}(k)$, form the set of the generalized hydrodynamic collective excitations. All the other eigenvalues correspond to kinetic modes, which in small- $k$ lim- it, in contrast to generalized hydrodynamic ones, have the finite damping coefficients (or finite lifetime) and do not contribute significantly to the long-wavelength dynamics. However, beyond the hydrodynamic region the real parts of generalized hydrodynamic and kinetic modes can have comparable values, and the relevant kinetic modes can change the hydrodynamic shape of time correlation functions. The approximate width of hydrodynamic region for liquid Cs, beyond which the nonhydrodynamic effects are not anymore negligible, can be roughly estimated as the width of propagation gap $k_{H}$ for shear waves (transverse acoustic-like excitations). In Ref. [13] we have found $k_{H}$ to be $0.092 \AA^{-1}$.

Two branches of kinetic propagating modes $z_{2}^{ \pm}(k)$ and $z_{3}^{ \pm}(k)$ have much larger damping coefficients (real parts of eigenvalues in Figure 1b) than the generalized acoustic excitations in small- $k$ region. However, for $k>1 \AA^{-1}$ all the propagating excitations have comparable damping, that implies comparable contributions to the dynamical properties in that $k$-region. An interesting feature is that the branch $z_{2}^{ \pm}(k)$ exhibits for small wavenumbers rapidly decaying behaviour, what makes sense to study more carefully the origin of this propagating modes as well as its contribution to the density-density time correlation functions.

Qualitatively the nine-mode spectrum of generalized collective excitations shown in Figures 1 and 2 for $k<$ $2 \AA^{-1}$ is similar to the case of liquid Bi [18]. For higher $k$-values, however, it resembles the one obtained in the same approximations for a Lennard-Jones fluid [17] and liquid $\mathrm{Pb}[30]$, where a low-frequency branch of heat waves with a relatively small propagation gap was found. The emergence of the low-frequency branch $z_{0}^{ \pm}(k)$ complicates very much the understanding of general features of mode contributions to the time correlation functions. Therefore, we will mainly focus in this study on the physical origin of mode formation and the calculations of mode contributions to time correlation functions of a liquid Cs in the region of propagation gap $\left(k<2.2 \AA^{-1}\right)$ for the low-frequency branch $z_{0}^{ \pm}(k)$.

\section{B. Analysis of the origin of collective excitation branches}

For the correct interpretation of generalized collective excitations one has to investigate carefully main physical processes responsible for the mode formation. In this study, we proceed in the following steps: i) we introduce the separated orthogonal subsets of dynamical variables, which describe some 'pure' physical processes being strictly identified as thermal or/and viscoelastic ones; ii) the spectra of generalized modes are calculated on the separated subsets, generated by these new orthogonal dynamical variables; iii) comparing the results, obtained for the spectra on the separated subsets as well as on the basis set $\mathbf{A}^{(9)}$, one can establish in a systematic way which kind of processes are mainly responsible for an appearance of each branch in the spectrum. In the case of week mode coupling effects we may expect, that 
some collective modes, obtained for the 'coupled' basis set $\mathbf{A}^{(9)}$, will be reproduced very well by the eigenvalues found for the separated subsets. The similar analysis has been used previously in our studies of the longitudinal dynamics of liquid $\mathrm{Pb}[30]$ and $\mathrm{Bi}$ [18] and the transverse dynamics of binary liquids [24]. In the later case, in particular, it was proved the existence of optic-like excitations in nonionic binary liquids [24] under some special condition.

It is well known that in contrast to the energy density operator, one can consider the operator $\hat{h}(k, t)$,

$$
\hat{h}(k, t)=\hat{e}(k, t)-\frac{f_{n e}(k)}{f_{n n}(k)} \hat{n}(k, t),
$$

which is orthogonal to the particle density operator $\hat{n}(k, t)$. In (9) the $f_{n e}(k)$ and $f_{n n}(k)$ are the static correlation functions 'density-energy' and 'densitydensity' [or static structure factor $f_{n n}(k) \equiv S(k)$ ], respectively. Hence, the hydrodynamic set of three variables $\hat{n}(k, t), \hat{J}(k, t), \hat{h}(k, t)$ contains only the orthogonal dynamic variables, and this set can be extended by their time derivatives within the generalized collective mode approach. The dynamical variable $\hat{h}(k, t)$ describes, in fact, the heat density fluctuations $[29,36]$, and in the limit $k \rightarrow 0$ it is easily to show that the thermodiffusive mode (8) emerges exclusively due to the heat density fluctuations.

Let us consider now the two separated sets of dynamical variables, which describe separately the heat and viscoelastic processes, namely:

$$
\mathbf{A}^{(4 h)}(k, t)=\{h(k, t), \dot{h}(k, t), \ddot{h}(k, t), \dddot{h}(k, t)\}
$$

and,

$$
\mathbf{A}^{(5 v)}(k, t)=\left\{n(k, t), J_{l}(k, t), \dot{J}_{l}(k, t), \ddot{J}_{l}(k, t), \dddot{J}_{l}(k, t)\right\} .
$$

Both subsets (10) and (11) together form the ninevariable basis set, which could be related to $\mathbf{A}^{(9)}(k, t)$ [see (1)] by simple linear transformation of the dynamic variables. Note that in general it is easily to check out that a linear transformed set and an initial one should have the same set of eigenvalues. Hence, we can expect that the spectra of collective modes, obtained on the separated subsets (10) and (11), should be very similar to the results obtained previously (see Figures 1 and 2) and the comparison of spectra gives us an additional information about the origin of modes formation and the strength of mode coupling effects.

In Figure 3 the spectra of propagating eigenvalues, obtained on the separated sets (10) and (11), are shown by dashed and solid spline lines, respectively. It is well seen, that the second branch of propagating modes $z_{2}^{ \pm}(k)$ reflects the heat density fluctuations in liquid. The highfrequency branch of propagating modes $z_{3}^{ \pm}(k)$ is also con- nected with the thermal processes and due to the high damping can not contribute significantly to the dynamical properties of a liquid Cs in small- $k$ region. For small wavenumbers, these excitations have extremely short lifetime, defined as the inverse real part of their eigenvalues, in comparison with the hydrodynamic modes. One can say also, that in the region $k>0.4 \AA^{-1}$ the heat and viscoelastic processes are well separated and the subsets (10) and (11) generate eigenvalues in very nice agreement with the nine-variable spectrum, shown by symbols. This finding is in agreement with the conclusion, made in Ref. [3], that the anomalous dispersion of sound excitations in a liquid $\mathrm{Cs}$ near the melting point can mainly be explain by the viscoelastic mechanism of the Maxwell-like relaxation. For smaller wavenumbers it is seen in Figure 3 that the mode coupling effects between the heat and viscoelastic processes increase and that results in the observed deviation of the values found for the branch $z_{2}^{ \pm}(k)$ on two different sets of dynamic variables, namely, on the sets (10) and (1).

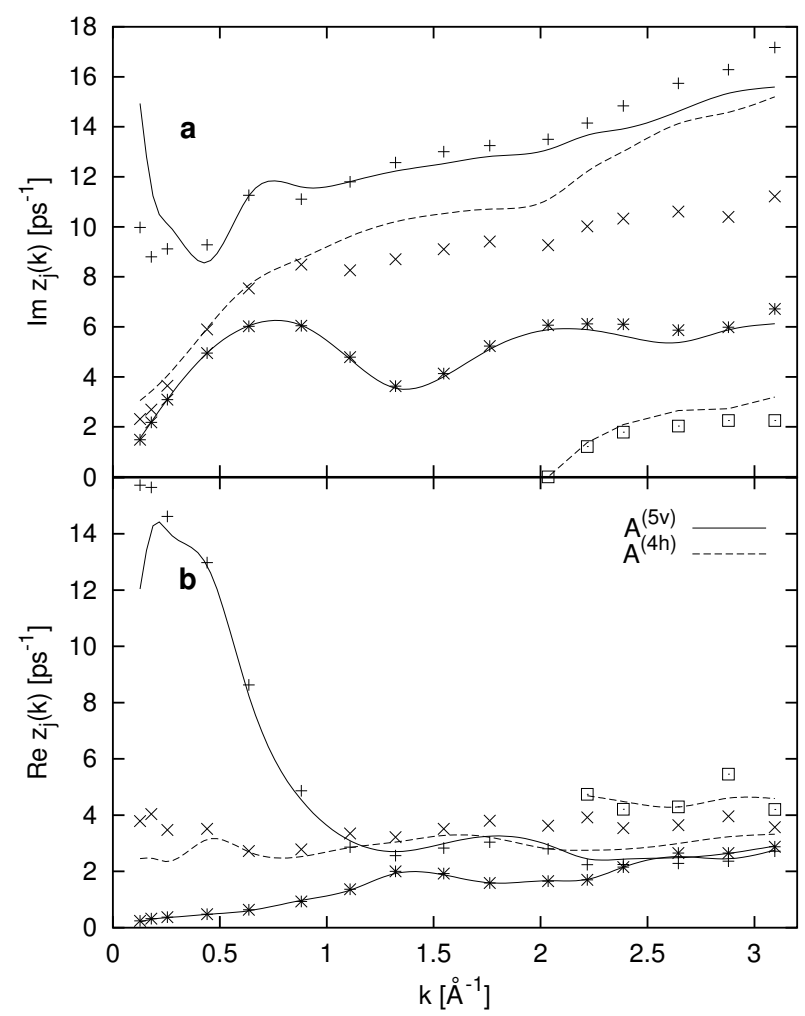

Fig. 3. Spectra of propagating collective excitations, obtained for the separated subsets $\mathbf{A}^{(5 v)}(k, t)$ (spline interpolated solid line) and $\mathbf{A}^{(4 h)}(k, t)$ (spline interpolated dashed line). Imaginary and real parts of eigenvalues are shown in the frames (a) and (b), respectively. Symbols denote the same eigenvalues as in Figure 1.

The four-variable subset $\mathbf{A}^{(4 h)}(k, t)$, describing the separated heat fluctuations, yields a pair of complex conjugated eigenvalues $z_{2}^{ \pm}(k)$ and two purely real modes $\left[d_{1}(k)\right.$ and $\left.d_{3}(k)\right]$, when $k$ is small $\left(k<2.2 \AA^{-1}\right)$. A general tendency for the $k$-dependence of purely relaxing eigenvalues $d_{1}(k)$ and $d_{3}(k)$ is to have closer values, when 
$k$ increases to the region of emergence of a new propagating branch [heat waves $\left.z_{0}^{ \pm}(k)\right]$. For higher $k$-values instead of the pair of purely relaxing modes one obtains an additional pair of complex-conjugated eigenvalues, which correlate well with the branch $z_{0}^{ \pm}(k)$ shown in Figure 1. Similar behaviour of relaxing modes in the region of the emergence of new branch of propagating excitations was observed in the case of transverse dynamics [23]. The analogy between shear waves and low-frequency kinetic heat waves was used in our analytical treatment of heat waves for the case of a liquid $\mathrm{Pb}$, reported in [30].

In small $k$-domain the lowest lying purely real eigenvalue, obtained on the subset $\mathbf{A}^{(4 h)}(k, t)$ (see the dashed line in Figure 4), correlates very well with the thermodiffusive mode (8), obtained on the nine-variable basis set (1) and marked by us in Figure 2 as $d_{1}(k)$. For larger wavenumbers (starting from $k \sim 0.3 \AA^{-1}$ ), due to the strong coupling of the modes $d_{1}(k)$ and $d_{2}(k)$, one can see some deviation between these purely real eigenvalues, obtained on the four- (10) and nine-variable (1) basis sets. Another interesting feature that follows from our study is seen in Figure 2: the thermodiffusive heat mode $d_{1}(k)$ is not in fact the lowest one in the nine-mode spectrum for the intermediate and large wavenumbers. In this region the significant contribution to time correlation functions can be expected from another relaxing mode $d_{2}(k)$, which is mainly caused by the viscoelastic processes.

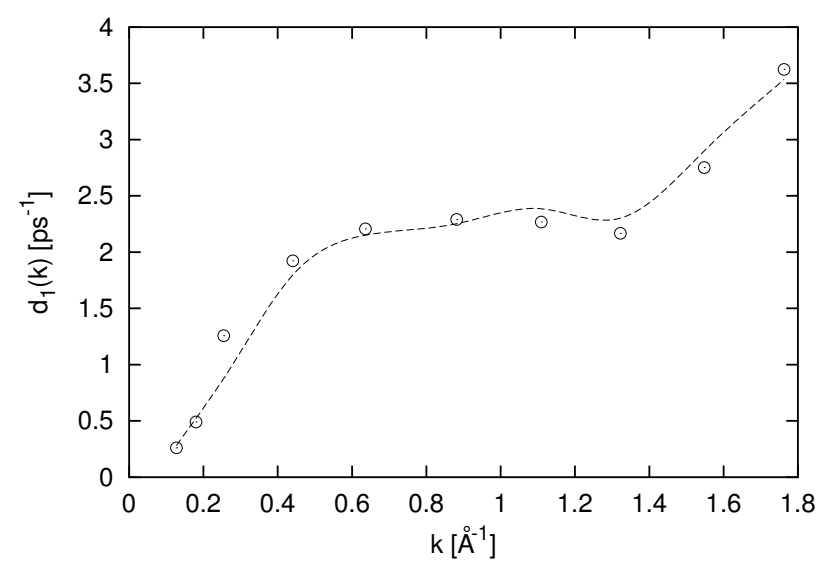

Fig. 4. Relaxing mode $d_{1}(k)$ obtained for the nine-variable set $\mathbf{A}^{(9)}(k, t)$ (open circles) and the lowest relaxing mode found for the four-variable set $\mathbf{A}^{(4 h)}(k, t)$ (spline interpolated dashed line). Symbols denote the same eigenvalues as in Figure 2.

The viscoelastic subset $\mathbf{A}^{(5 v)}(k, t)$ generates five eigenvalues, namely, two pairs of propagating modes, which correspond to $z_{1}^{ \pm}(k)$ and $z_{3}^{ \pm}(k)$, and one relaxing kinetic mode $d_{2}(k)$. In Figure 5 the eigenvalue $d_{2}(k)$, obtained for the 'coupled' nine-variable basis set and shown by closed boxes, is given in comparison with the results, found for the five-variable subset $\mathbf{A}^{(5 v)}(k, t)$ (solid line). It is well seen that for $k>0.3 \AA^{-1}$ both $k$-dependences are in very good correlation. Otherwise, for smaller wavenumbers a rather strong deviation between the rel- evant relaxing eigenvalues, obtained by the 'coupled' basis set $\mathbf{A}^{(9)}(k, t)$ and the viscoelastic one $\mathbf{A}^{(5 v)}(k, t)$, is observed. This indicates, that the viscoelastic approach cannot reproduce correctly the collective relaxing behaviour close to the hydrodynamic region. Another interesting conclusion can be made by comparing behaviour of $d_{2}(k)$ with a relaxing mode, obtained within the simplest one-variable treatment with $\mathbf{A}^{(1)}(k, t)=\{\hat{n}\}$. One can immediately derive the analytic expression for this single-mode eigenvalue:

$$
d_{0}(k)=\tau_{n n}^{-1}(k)
$$

where $\tau_{n n}(k)$ is the generalized correlation time associated with 'density-density' time correlation function [15]. It is seen in Figure 5 that the eigenvalue $d_{0}(k)$ (dashed line) qualitatively behaves very similar to $d_{2}(k)$. Moreover, it reproduces perfectly the behaviour of $d_{2}(k)$ (closed boxes) in the region of $k \sim k_{\mathrm{p}}$, where the main peak of static structure factor is located. This means, that in this $k$-region the relaxing kinetic mode $d_{2}(k)$ is completely defined by the density fluctuations, which are well separated from the other viscoelastic processes associated with the current operator $\hat{J}_{l}(k, t)$ and its time derivatives. Similar result was obtained previously in the case of a liquid $\mathrm{Pb}[30]$ for the state near the melting point and this seems to be a quite general feature in a simple liquid.

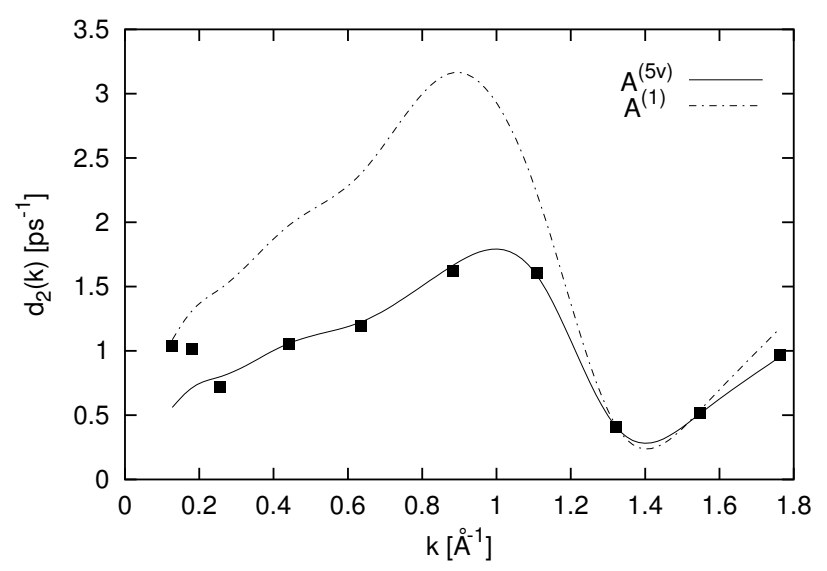

Fig. 5. Relaxing mode $d_{2}(k)$ obtained for the nine-variable set $\mathbf{A}^{(9)}(k, t)$ (closed boxes), the only relaxing mode found for the five-variable set $\mathbf{A}^{(5 v)}(k, t)$ (spline interpolated solid line) and the eigenvalue obtained for the single-variable set $\mathbf{A}^{(1)}(k, t)$. Symbols denote the same eigenvalues as in Figure 2 .

\section{Mode contributions to time correlation functions}

The GCM method with the basis set of $N_{v}$ dynamic variables allows one to separate the $N_{v}$ mode contributions to different time correlation functions, constructed on the variables considered, as well as to any corresponding spectral functions, according to (2) and (3), respec- 
tively. In this study we are mainly interested in calculations of the mode contributions, caused by the generalized collective modes, to the 'density-density' and 'heat density-heat density' time correlation functions.

The 'density-density' time correlation function. In Figures $6 \mathrm{a}$ and $6 \mathrm{~b}$ the separated mode contributions to the 'density-density' time correlation function, obtained at two values of $k$, are shown by different lines. It is seen in Figure 6a that at the smallest wavenumber $k=k_{\text {min }}$, sampled in our MD simulations, the shape of $F_{n n}(k, t)$ (solid line) is mainly determined by the sound excitations $z_{1}^{ \pm}(k)$ (short-dashed line), and, as it could be shown, the symmetric contribution (see Eq. 5) of these modes is dominant. The contribution of heat waves $z_{2}^{ \pm}(k)$ (long-dashed line) is smaller in several orders of magnitude. Two relaxing modes also contribute to the function $F_{n n}(k, t)$ for this $k$-value. One can distinguish in Figure $6 \mathrm{a}$ the long-time contribution of thermodiffusive mode $d_{1}(k)$ (dotted line) and short-time one of kinetic relaxing mode $d_{2}(k)$ (dash-dotted line). Taking into account the relatively large magnitude of kinetic relaxing mode $d_{2}(k)$, one can conclude that in fact the wavenumber $k=k_{\text {min }}$ considered is already beyond the hydrodynamic region. This is in agreement with our estimates found in the study of transverse dynamics [13].

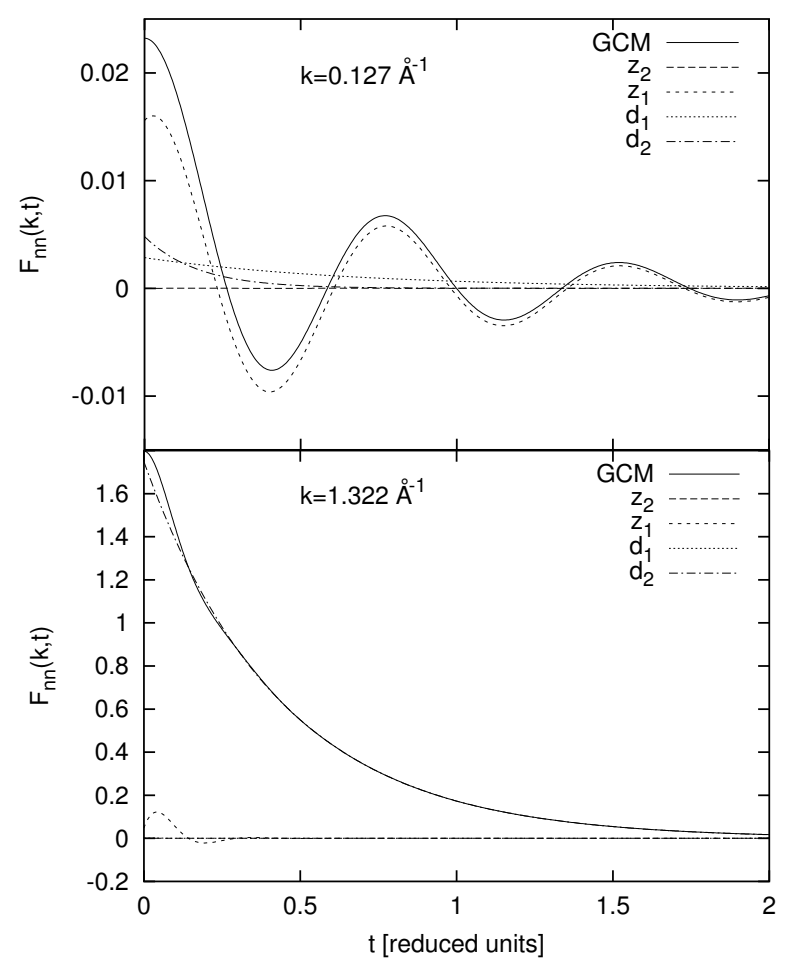

Fig. 6. Mode contributions to the 'density-density' time correlation functions for two $k$-values: (a) $k=0.127 \AA^{-1}$ and (b) $k=1.322 \AA^{-1}$. Time scale is $5.6626 \mathrm{ps}$.

At higher value of wavenumber, $k=1.322 \AA^{-1}$, two main contributions to the 'density-density' time correlation function are seen in Figure $6 \mathrm{~b}$. The tail of time correlation function is completely defined by the kinet- ic relaxing mode $d_{2}(k)$ (dash-dotted line) and a small short-time contribution is due to the acoustic-like modes $z_{1}^{ \pm}(k)$ (it was found that this is mainly an asymmetric contribution). Another interesting finding is that for the $k$ considered the thermodiffusive mode $d_{1}(k)$ does not make any visible contribution to the shape of $F_{n n}(k, t)$.

In more complete form the study of mode contributions can be presented by considering the $k$-dependence of the normalized [with the factor $1 / F_{n n}(k, 0)$ ] amplitudes $\bar{A}_{n n}^{r}(k), \bar{B}_{n n}^{p}(k)$ and $\bar{C}_{n n}^{p}(k)$ (see Eq. $\left.(5)\right)$. In Figure $7 \mathrm{a}$ the normalized mode amplitudes of some generalized collective excitations are shown, namely, we have plotted in this Figure: the symmetric $\bar{B}^{1}$ (open circles) and asymmetric $\bar{C}^{1}$ (closed circles) contributions of the sound excitations $z_{1}^{ \pm}(k)$; the symmetric $\bar{B}^{2}$ (open triangles) contribution of the propagating kinetic modes $z_{2}^{ \pm}(k)$; and the normalized amplitudes $\bar{A}^{1}$ (asterisks) and $\bar{A}^{2}$ (closed boxes) of the thermodiffusive $d_{1}(k)$ and kinetic relaxing $d_{2}(k)$ modes, respectively. It is seen in the Figure 7 a that the generalized thermodiffusive mode $d_{1}(k)$ only weakly contributes to $F_{n n}(k, t)$, when $k>0.4 \AA^{-1}$. In the hydrodynamic limit $k \rightarrow 0$ its amplitude tends to the value $(\gamma-1) / \gamma$, predicted by the linear hydrodynamics $[35,36]$. In the case of a liquid Cs an experimental value of the ratio of specific heats is $\gamma=1.102[4,12]$, and the normalized amplitude of thermodiffusive contribution in the hydrodynamic limit has to reach the value 0.093. From the other side, it is well-known $[35,36]$ that, according to hydrodynamic expressions, the amplitude of generalized sound excitations $z_{1}^{ \pm}(k)$ should tend to the value $1 / \gamma=0.907$. One can see in Figure $7 \mathrm{a}$, that when $k \rightarrow 0$, the symmetric contribution of generalized acoustic modes is rapidly increasing. However, for the smallest wavenumber, considered in our MD simulations, only the tendency to reach the appropriate hydrodynamic values is observed for both amplitudes $\bar{A}^{1}$ and $\bar{B}^{1}$. In this respect we would like to remind, that the width of hydrodynamic region for a liquid Cs near the melting point is very small (at least smaller than $0.092 \AA^{-1}$ as it was shown in [13]).

The normalized symmetric amplitudes of kinetic heat waves $z_{2}^{ \pm}(k)$ are shown by triangles in Figure 7a. It is seen, that these excitations almost do not contribute to the 'density-density' time correlation function. Nevertheless, there exists another kinetic mode, namely the relaxing mode $d_{2}(k)$ (closed boxes), the normalized amplitude of which is rather large beyond the hydrodynamic region. If $k \rightarrow 0$ this amplitude tends to zero (as it should be according the linear hydrodynamic theory), but in the region $0.4 \AA^{-1}<k<1.1 \AA^{-1}$ the kinetic relaxing mode $d_{2}(k)$ contributes sufficiently with the amplitude comparable with the symmetric contribution of generalized sound excitations $z_{1}^{ \pm}(k)$ (open circles). In the region close to $k_{\mathrm{p}}$ the kinetic relaxing mode $d_{2}(k)$ defines almost completely the shape of 'density-density' time correlation functions. Otherwise, as far as we can judge from Figure $7 \mathrm{a}$, in the region of $k \sim k_{\mathrm{p}}$, the symmetric contribution of acoustic excitations is extremely small. This, in particular, explains well why numerous attempts to find far beyond the hydrodynamic region a sufficient ev- 
idence for the propagation of sound modes with the help of simple methods, based on spectral analysis scheme alone, were mainly not very successful (see, e.g., [10] and the references cited herein). Observing the results presented in Figure 7a, we can conclude that absence of a visible side sound peak in the dynamic structure factor does not mean the absence of generalized sound modes. In many cases such a peak is impossible to observe just because of small relative symmetric amplitude of sound excitations, what is well seen in fact in Figure $7 \mathrm{a}$ for $0.85 k_{\mathrm{p}}<k<1.15 k_{\mathrm{p}}$. In this range of wavenumbers there is also a small asymmetric contribution [associated with the non-Lorentzian term in (5)] from the generalized sound modes with the normalized amplitude (closed circles) being about 0.2 in the whole $k$-region considered.
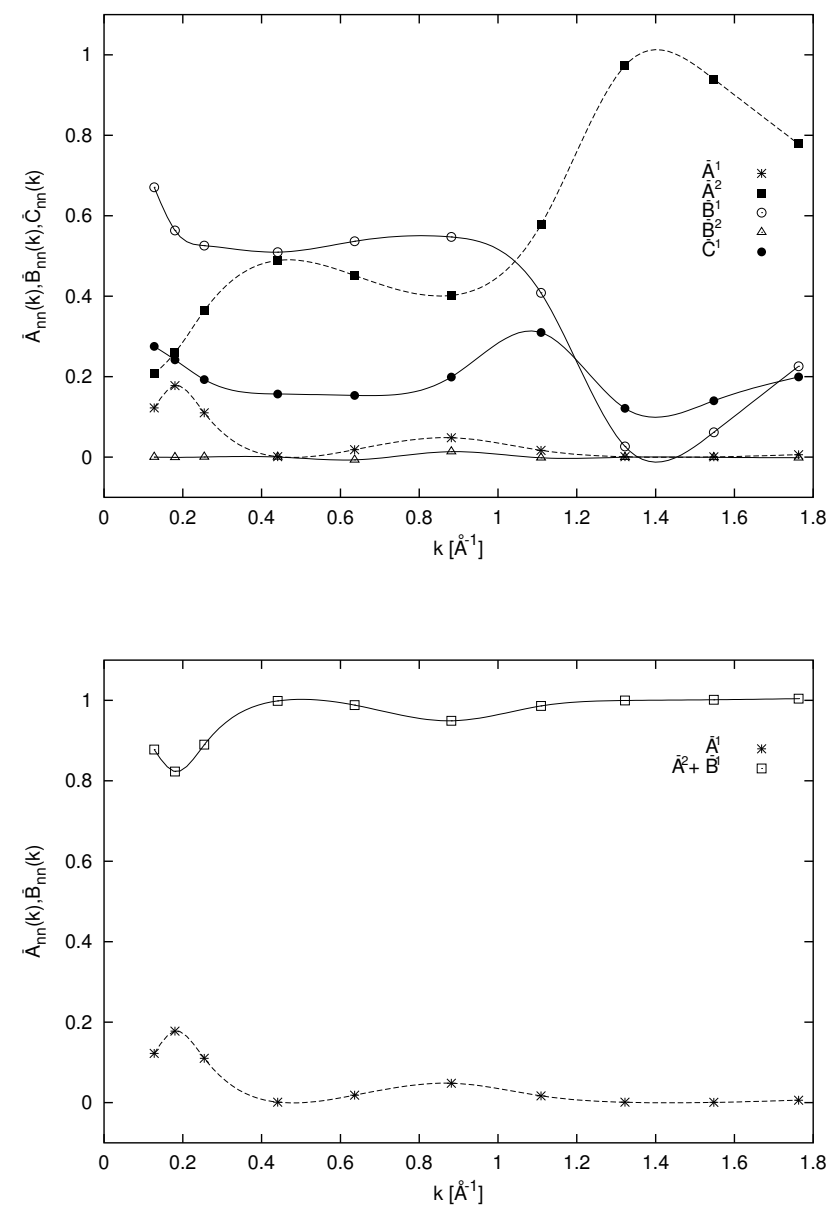

Fig. 7. Normalized amplitudes of mode contributions to the 'density-density' time correlation function: (a) relaxing modes $d_{1}(k)$ (asterisks) and $d_{2}(k)$ (closed boxes), generalized sound excitations $z_{1}^{ \pm}(k)$ (symmetric and asymmetric contributions are shown by open and closed circles, respectively), and heat waves $z_{2}^{ \pm}(k)$ (symmetric contribution, open triangles); (b) The sum of symmetric contribution from the sound excitations and contribution from relaxing kinetic mode $d_{2}(k)$ (open boxes). Lines denote spline interpolation.

Another conclusion, which follows from the results presented in Figure 7a, is that in a wide range of wavenumbers the shape of function $F_{n n}(k, t)$ is mainly determined by the viscoelastic properties. In order to illustrate this statement we have plotted in Figure $7 \mathrm{~b}$ the sum of two normalized amplitudes, namely, the amplitudes describing the symmetric contribution $\bar{B}^{1}$ of sound excitations $z_{1}^{ \pm}(k)$ and the contribution $\bar{A}^{2}$ of the kinetic relaxing mode $d_{2}(k)$. The function $\bar{A}_{n n}^{1}(k)$, which gives the information about the relative weight of thermal processes, is also shown in Figure 7b. It is well seen, that just beyond a small- $k$ region (in our case for $k>0.4 \AA^{-1}$ ), in which the contribution of heat processes is of the order predicted by the hydrodynamic theory, the shape of 'densitydensity' time correlation function is almost completely defined by the viscoelastic processes. In particular, this means, that for larger $k$, in contrast to the predictions of the purely hydrodynamic treatment, the central peak of dynamic structure factor $S(k, \omega)$ can not be attributed anymore to the thermodiffusion and is mainly formed by the viscoelastic processes. One of the evident reason for such a result is a small value of $(\gamma-1) \simeq 0.1$ in the system considered, showing that the static coupling between the thermal and viscoelastic properties is rather weak. The conclusion drawn allows one to understand better the success of different viscoelastic theories (see, e. g., $[3,4,10,11])$, used for the description of dynamic structure factor in a liquid Cs. It is also seen in Figure $7 \mathrm{~b}$ that when the wavenumber $k$ decreases, the sum of contributions $\bar{A}^{2}$ and $\bar{B}^{1}$ as a function of $k$ behaves like the symmetric contribution of sound modes alone. This just reflects the fact that the amplitude $\bar{A}^{2}$ of relaxing kinetic mode $d_{2}(k)$ is decreasing at least as $k^{2}$ when $k$ is small. However, as it was already mentioned above, the contribution of this mode becomes very sufficient for intermediate and large wavenumbers.

In order to clarify further the physical meaning of relaxing kinetic mode $d_{2}(k)$, let us recall the expression (6) for zeroth order frequency moment and apply this expression to the function $F_{n n}(k, t)$. Taking into account, that the contribution of the mode $d_{2}(k)$ to $F_{n n}(k, t)$ is dominant at $k \sim k_{\mathrm{p}}$ (see Figure 7a) one may conclude that the kinetic relaxing mode defines almost completely the main peak of the static structure factor $S(k)$ in the sense of Eq. (6) [or vice versa]. This means that the kinetic mode $d_{2}(k)$ reflects mainly the structural properties of a liquid [at least on the spatial scale of nearest neighbors] and, therefore, one can call $d_{2}(k)$ as the structural relaxation kinetic mode. Following along such a line it is possible to distinguish three $k$-regions, in which the shape of static structure factor $S(k)$ is related to different kinds of dynamical processes: (i) in the longwavelength limit the sound excitations and thermodiffusion process are dominant, (ii) for intermediate $k$-values only the acoustic modes and the kinetic structural relaxation contribute to $S(k)$, and (iii) when $k \sim k_{\mathrm{p}}$ the kinetic structural relaxation is mainly responsible for the structural properties and the shape of the main peak of $S(k)$.

The 'heat density-heat density' time correlation function. Completely opposite picture for the mode contributions is observed in the case of the 'heat density-heat density' time correlation function (see Figures $8 \mathrm{a}$ and $8 \mathrm{~b})$. For the smallest $k$-value considered in our MD sim- 
ulations, the shape of the function $F_{h h}(k, t)$ is mainly determined by the thermodiffusive mode $d_{1}(k)$ (dotted line in Figure 8a). The generalized sound excitations $z_{1}^{ \pm}(k)$ (short-dashed line) make rather small and mainly symmetric contribution which results in some modulation of $F_{h h}(k, t)$. There exist also very small short-time contributions of the high-frequency heat waves $z_{2}^{ \pm}(k)$ (long-dashed line) as well as the kinetic relaxing mode $d_{2}(k)$ (dash-dotted line). For larger wavenumber, $k=$ $1.322 \AA^{-1}$, only two modes determine mainly (see Figure $8 \mathrm{~b})$ the shape of $F_{h h}(k, t)$, namely, the thermodiffusive mode $d_{1}(k)$ and the high-frequency heat waves $z_{2}^{ \pm}(k)$. In the later case the asymmetric contribution is dominant.

The linear hydrodynamic theory predicts for the time correlation function $F_{h h}(k, t)[35,36]$, that the normalized amplitude of thermodifusive mode $d_{1}(k)$ and the symmetric normalized contribution of sound excitations $z_{1}^{ \pm}(k)$ should be $1 / \gamma$ and $(\gamma-1) / \gamma$, respectively. It is seen in Figure 9 that at the smallest wavenumber $k_{\min }$ considered, these amplitudes (shown by asterisks and open circles, respectively) are already very close to the values, predicted by the hydrodynamic theory. For $k>0.4 \AA^{-1}$ the contributions of generalized sound excitations into the 'heat density-heat density' time correlation function are very small. The normalized amplitude of thermodiffusive mode $\bar{A}_{h h}^{1}(k)$ exhibits a minimum for $k \sim 0.3 \AA^{-1}$, originated by strong hybridization effects between two relaxing modes $d_{1}(k)$ and $d_{2}(k)$ with a cross-section (see Figure 2) in this $k$-region.
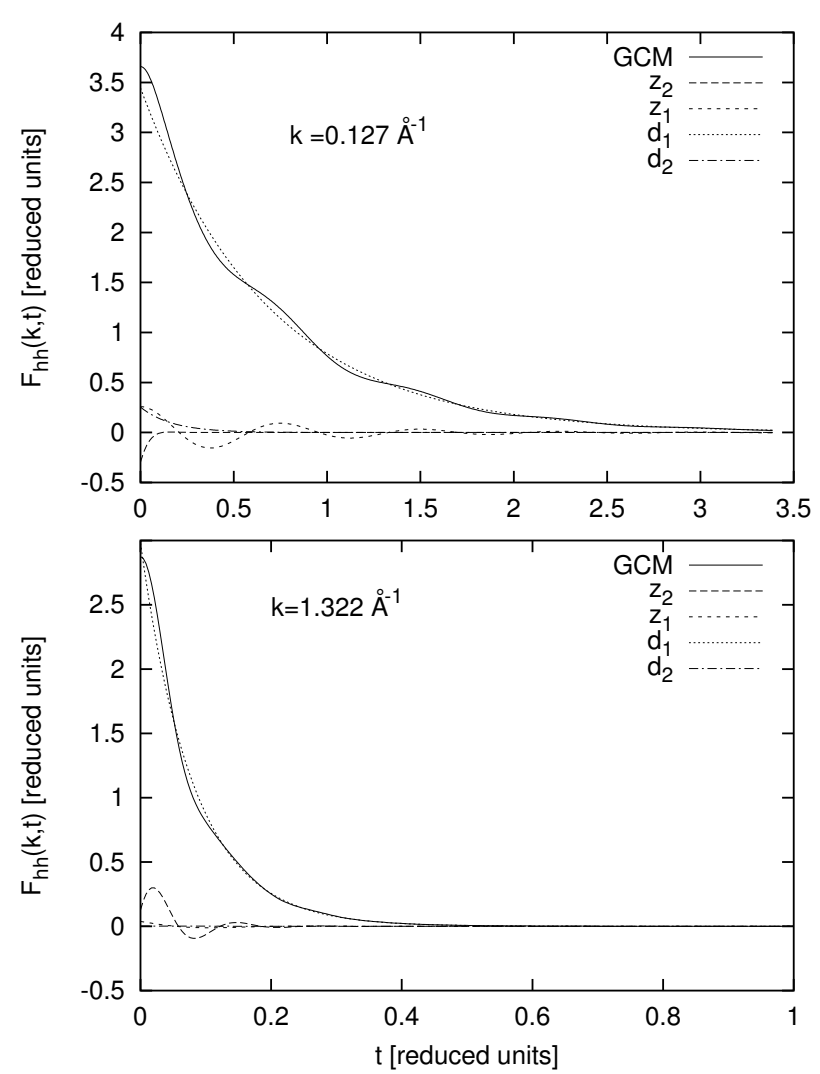

Fig. 8. Mode contributions to the 'heat density-heat density' time correlation function for two $k$-values: (a) $k=0.127 \AA^{-1}$ and (b) $k=1.322 \AA^{-1}$. Time scale is $5.6626 \mathrm{ps}$.
For intermediate $k$-values the generalized thermodiffusive mode $d_{1}(k)$ determines almost completely the shape of $F_{h h}(k, t)$. Interesting to note, that for wavenumbers approaching the boundary of propagation gap for the low-frequency heat waves $z_{0}^{ \pm}(k)$ (see Figure 1a) the normalized amplitudes of relaxing modes $d_{1}(k)$ and $d_{3}(k)$ (shown by open boxes in Figure 9 ) take rather large positive and negative values, respectively. However, it can be shown easily that in the vicinity of propagation gap boundary the sum of these contributions is finite, so that all the properties of time correlation function $F_{h h}(k, t)$ are reproduced correctly. It is also seen in Figure 9 that the high-frequency heat waves $z_{2}^{ \pm}(k)$ contribute weakly to the shape of $F_{h h}(k)$ (symmetric and asymmetric contributions are shown by open and closed triangles, respectively). Thus, one can make a conclusion, that in the case of 'heat density-heat density' time correlation function the main relaxing contribution for the system considered is due to the generalized thermodiffusive mode $d_{1}(k)$.

It should be noted that the results for the normalized amplitudes, presented in this subsection, together with the calculations, performed for the generalized collective modes, gives us the full information about the 'densitydensity' and 'heat density-heat density' time correlation functions in the considered range of $k$ as well as about other time correlation functions which can be written as their time derivatives (see (1) and (2)).

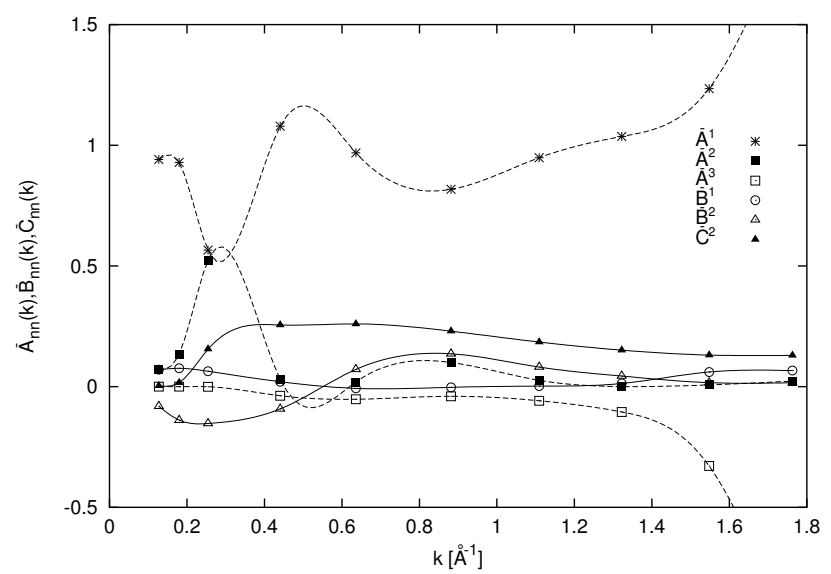

Fig. 9. Normalized amplitudes of mode contributions to the 'heat density-heat density' time correlation function: (a) Relaxing modes $d_{1}(k)$ (asterisks) and $d_{2}(k)$ (closed boxes), $d_{3}(k)$ (open boxes), generalized sound excitations $z_{1}^{ \pm}(k)$ (symmetric contribution, open circles), and the high-frequency heat waves $z_{2}^{ \pm}(k)$ (symmetric and asymmetric contributions are shown by open and closed triangles, respectively).

\section{CONCLUSIONS}

The main results of this study are as follows:

(i) The spectrum of generalized collective excitations 
of a liquid metallic Cs near the melting point is obtained and analyzed within the nine-variable GCM approach. These results are found in self-consistent way without any adjustable or fitting parameters. With the help of an idea of separated subsets of dynamic variables we have studied in detail the origin of mode formation that allows us to identify precisely each branch in the spectrum of longitudinal collective excitations. In particular, it is shown that in the system considered the truly hydrodynamic region is very narrow and in a wide $k$-range beyond this region the dynamical processes, describing the heat and viscoelastic fluctuations, are well separated;

(ii) It is shown that one of the kinetic relaxing modes found, namely the mode marked $d_{2}(k)$, plays a very important role in the dynamics of density fluctuations. In particular, the contribution of this mode to 'densitydensity' time correlation function $F_{n n}(k, t)$ in the region of intermediate wavenumbers (close to the position of the first maximum of static structure factor) is dominant and, therefore, this mode might be considered as responsible for structural relaxation phenomena. Otherwise, the generalized sound excitations contribute to $F_{n n}(k, t)$ very weakly in this range, so that it might be a good example when the well defined excitations are not visible, however, in time correlation function because of extremely small amplitudes of their contributions;

(iii) On the basis of our numerical study of the mode contributions to the 'density-density' and 'heat density- heat density' time correlation functions one can conclude that in the system considered the thermal and viscoelastic fluctuations are rather well separated beyond the hydrodynamic region: the relaxing behaviour of 'density-density' time correlation function for intermediate wavenumbers is mainly determined by structural relaxation, whereas the 'heat density-heat density' correlation function reflects mostly the processes, described by the generalized thermodiffusive mode.

We would like finally to make a remark, that this GCM study of dynamics of a liquid metal above the melting point is based on MD simulations with two-body interatomic potentials, which allow us to reproduce very nicely a static structure factor $S(q)[4,12]$. Therefore, we are not able to study bonding correlations in systems above freezing, which can affect the spectrum of collective excitations. Such a study is possible to perform by combining the GCM method with ab initio MD simulations, that might be the next step in applications of the GCM approach.

\section{ACKNOWLEDGEMENT}

I. M. thanks Fond für Förderung der Wissenschaftlichen Forschung, Austria, Project No. P15247 for support.
[1] N. H. March, Liquid Metals. Concepts and Theory (University Press, Cambridge, 1990).

[2] T. Bodensteiner, C. Morkel, W. Gläser, B. Dorner, Phys. Rev. A 45, 5709 (1992); T. Bodensteiner, Thesis TU München (1990).

[3] Chr. Morkel, T. Bodensteiner, H. Gemperlein, Phys. Rev. E 47, 2575 (1993).

[4] S. Kambayashi, G. Kahl, Phys. Rev. A 46, 3255 (1992).

[5] U. Balucani, A. Torcini, R. Vallauri, Phys. Rev. A 46, 2159 (1992).

[6] U. Balucani, A. Torcini, R. Vallauri, Phys. Rev. B 47, 3011 (1993).

[7] D. L. Price, K. S. Singwi, M. P. Tosi, Phys. Rev. B 2, 2983 (1970).

[8] S. Kambayashi, Y. Hiwatari, Phys. Rev. E 49, 1251 (1994).

[9] M. Canales, J. A. Padro, Phys. Rev. E 60, 551 (1999).

[10] R. K. Sharma, K. Tankeshwar, Phys. Rev. E 55, 564 (1997).

[11] R. M. Yulmetyev, A. V. Mokshin, P. Hänggi, V. Yu. Shurygin, Phys. Rev. E 64, 057101 (2001).

[12] T. Bryk, Y. Chushak, J. Phys.: Condens. Matter 9, 3329 (1997).

[13] T. Bryk, I. Mryglod, J. Phys. Stud. 2, 322 (1998).

[14] I. M. de Schepper, E. G. D. Cohen, C. Bruin, J. C. van Rijs, W. Montfrooij, L. A. de Graaf, Phys. Rev. A 38, 271 (1988).

[15] I. M. Mryglod, I. P. Omelyan, M. V. Tokarchuk, Mol.
Phys. 84, 235 (1995).

[16] I. M. Mryglod, Condens. Matter Phys. 1, 753 (1998).

[17] I. M. Mryglod, I. P. Omelyan, Phys. Lett. A 205401 (1995).

[18] T. Bryk, I. Mryglod, J. Phys.: Condens. Matter 12, 3543 (2000); J. Phys.: Condens. Matter 13, 1343 (2001).

[19] D. Bertolini, A. Tani, Phys. Rev. E 51, 1091 (1995).

[20] P. Westerhuijs, W. Montfrooij, L. A. de Graaf, I. M. de Schepper, Phys. Rev. A 45, 3749 (1992).

[21] T. Bryk, I. Mryglod, G. Kahl, Phys. Rev. E, 56, 2903 (1997).

[22] T. Bryk, I. Mryglod, J. Phys.: Condens. Matter 14, L445 (2002).

[23] T. Bryk, I. Mryglod, Phys. Rev. E, 62, 2188 (2000).

[24] T. Bryk, I. Mryglod, J. Phys.: Condens. Matter 12, 6063 (2000).

[25] T. Bryk, I. Mryglod, Phys. Lett. A 261, 349 (1999).

[26] T. Bryk, I. Mryglod, Condens. Matter Phys. 2, 285 (1999).

[27] J.-P. Boon, S. Yip, Molecular Hydrodynamics (McGrawHill, New-York, 1980).

[28] J.-P. Hansen, I. R. McDonald, Theory of Simple Liquids (Academic, London, 1986).

[29] U. Balucani, M. Zoppi, Dynamics of the Liquid State (Clarendon, Oxford, 1994).

[30] T. Bryk, I. Mryglod, Phys. Rev. E 63, 051202 (2001); Phys. Rev. E 64, 032202 (2001).

[31] I. M. Mryglod, I. P. Omelyan, Mol. Phys. 90, 91 (1997); 
Mol. Phys. 91, 1005 (1997); Mol. Phys. 92, 913 (1997).

[32] I. M. de Schepper, P. Verkerk, A. A. van Well, L. A. de Graaf, Phys. Lett. A 104, 29 (1984).

[33] I. Mryglod, J. Phys. Stud. 3, 33 (1999).

[34] R. Ohse, Handbook of Thermodynamic and Transport Properties of Alkali Metals (Blackwell Scientific, Oxford,
1985).

[35] C. Cohen, J. W. H. Sutherland, J. M. Deutch, Phys. Chem. Liq. 2, 213 (1971).

[36] N. H. March, M. P. Tosi, Atomic Dynamics in Liquids (Macmillan Press, London, 1976).

\section{УЗАГАЛЬНЕНІ КОЛЕКТИВНІ МОДИ В РІДКОМУ ЦЕЗІЇ}

Т. Брик, І. Мриглод

Інститут фізики конденсованих систем, Національна академія наук Украӥни

вул. Свениічького, 1, Львів, 79011, Украӥна

Спектр колективних збуджень у рідкому цезії при температурі $308 \mathrm{~K}$ досліджено в межах дев'ятизмінного наближення підходу узагальнених колективних мод. Застосовано оригінальний аналіз спектрів 3 метою визначення природи всіх отриманих гілок пропагаторних та релаксаційних колективних мод. Дві гілки низько- та високочастотних пропагаторних збуджень ідентифікуються з тепловими хвилями. Показано, що кінетична релаксаційна мода, породжена флюктуаціями густини, визначає майже повністю форму часових кореляційних функцій "густина-густина" для хвильових чисел, близьких до положення головного піка статичного структурного фактора. Докладно досліджено амплітуди модових внесків у часові кореляційні функції "густина-густина" та "теплова густина-теплова густина". 